\title{
Linear accelerator-based radiosurgery for vestibular schwannoma
}

\author{
William A. Friedman, M.D., AND Kelly D. Foote, M.D. \\ Department of Neurosurgery, University of Florida, Gainesville, Florida
}

\begin{abstract}
Despite major advances in skull base surgery and microsurgical techniques, surgery for vestibular schwannoma (VS) carries a risk of complications. Some are inherent to general anesthesia and surgery of any type and include myocardial infarction, pneumonia, pulmonary embolism, and infection. Some are specific to neurosurgery in this area of the brain, and include hydrocephalus, cerebrospinal fluid leak, facial nerve paralysis, facial numbness, hearing loss, ataxia, dysphagia, and major stroke. Even in the hands of very experienced acoustic surgeons, these risks cannot be eliminated.

Radiosurgery provides an outpatient, noninvasive alternative for the treatment of small acoustic schwannomas. Initially radiosurgery was undertaken in "high-risk" patients, including the elderly, those with severe medical comorbidities, and those in whom tumors recurred after surgery. Additionally, a high rate of cranial nerve morbidity was reported. With improvements in dosimetry planning and dose selection, however, authors practicing at radiosurgical centers now report very low complication rates, as well as high tumor control rates.

In this report the authors specifically review the results of linear accelerator-based radiosurgery for VS and compare these outcomes with the best surgical alternatives.
\end{abstract}

\section{KEY WoRdS - radiosurgery - linear accelerator • gamma knife • vestibular schwannoma • acoustic neuroma • complications}

Stereotactic radiosurgery is a minimally invasive treatment modality that allows delivery of a large single dose of radiation to a specific intracranial target while sparing surrounding tissue. Unlike conventional fractionated radiotherapy, stereotactic radiosurgery does not rely on, or exploit, the higher radiosensitivity of neoplastic lesions relative to normal brain (therapeutic ratio). Its selective destruction depends primarily on sharply focused highdose radiation and a steep dose gradient away from the defined target. The biological effect is irreparable cellular damage and delayed vascular occlusion within the high-dose target volume. Because a therapeutic ratio is not required, traditionally radioresistant lesions can be treated. Because destructive doses are used, however, any normal structure included in the target volume is subject to damage.

The basis for stereotactic radiosurgery was conceived more than 40 years ago by Lars Leksell. ${ }^{27} \mathrm{He}$ proposed the technique of focusing multiple nonparallel beams of external radiation on a stereotactically defined intracranial

Abbreviations used in this paper: $\mathrm{CT}=$ computerized tomography; GK = gamma knife; GKS = gamma knive surgery; LINAC = linear accelerator; $\mathrm{MR}=$ magnetic resonance; NF2 = neurofibromatosis Type 2; VS = vestibular schwannoma. target. The averaging of these intersecting beams results in very high doses of radiation being delivered to the target volume, while innocuously low doses reach nontargeted tissues along the path of any given beam. His team's implementation of this concept culminated in the development of the GK. The modern GK employs 201 fixed cobalt radiation sources in a fixed hemispherical array, such that all 201 photon beams are focused on a single point. The patient is stereotactically positioned in the GK so that the intracranial target coincides with the isocenter of radiation. Using variable collimation, beam blocking, and multiple isocenters, the radiation target volume is shaped to conform to the intracranial target.

An alternate radiosurgical solution, LINAC radiosurgery was first described in 1984 by Betti and Derechinsky. ${ }^{4}$ In 1985 , Colombo, et al., ${ }^{6}$ described such a system and LINACs have subsequently been modified in various ways to achieve the precision and accuracy required for radiosurgical applications. . $^{3,20,25,26,35,46,47,50,58,60}$ In 1986, a team composed of neurosurgeons, radiation physicists, and computer programmers began development of the University of Florida LINAC-based radiosurgery system. ${ }^{15}$ This system has been used to treat more than 2000 patients at the University of Florida since May 1988 and is in use at multiple sites worldwide. 
All LINAC radiosurgical systems rely on the same basic paradigm: a collimated x-ray beam is focused on a stereotactically identified intracranial target. The gantry of the LINAC rotates around the patient, producing an arc of radiation focused on the target (Fig. 1). The couch in which the patient rests is then rotated in the horizontal plane, and another arc is performed. In this manner, multiple noncoplanar arcs of radiation intersect at the target volume and produce a high target dose, resulting in minimal radiation affecting the surrounding brain. This dose concentration method is exactly analogous to the multiple intersecting beams of cobalt radiation in the GK.

The target dose distribution can be tailored by varying collimator sizes, eliminating undesirable arcs, manipulating arc angles, using multiple isocenters, and differentially weighting the isocenters. ${ }^{16}$ Achievable dose distributions are similar for LINAC-based and GK systems. With both systems, it is possible to deliver dose distributions that conform closely to the shape of the intracranial target, thus sparing the maximum amount of normal brain. Recent advances in stereotactic imaging and computer technology for dose planning, as well as refinements in radiation delivery systems, have led to improved efficacy, fewer complications, and a remarkable amount of interest in the various applications of stereotactic radiosurgery.

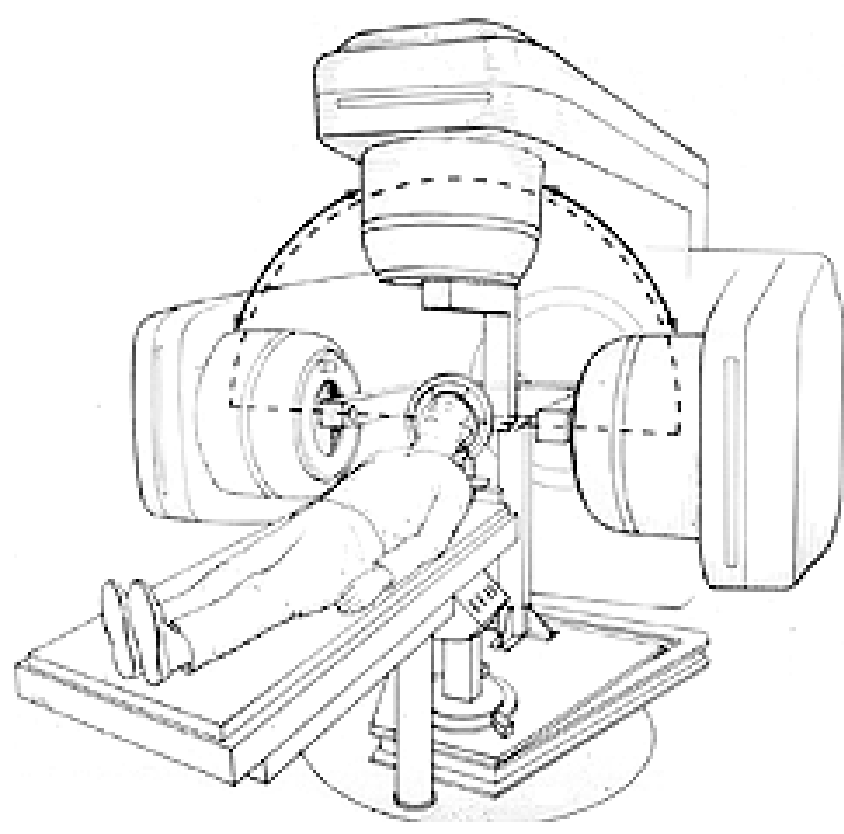

Fig. 1. A LINAC radiosurgery system. Linear accelerators are the preferred device, worldwide, for administering conventional radiotherapy. They accelerate electrons to near light speed, then collide them with a heavy metal (like tungsten) in the head of the machine. The collision mainly produces heat but a small percentage of the energy is converted into highly energetic photons. These photons, because they are electronically produced, are called " $\mathrm{x}$ rays." The radiation is collimated and focused on the target. This diagram shows an add-on device, designed to improve the accuracy of the LINAC, in place. The LINAC arcs around the patient, with its beam always focused on the stereotactically positioned target. The patient is then moved to a new horizontal (table) position, and another is performed. The result is multiple, noncoplanar arcs of radiation, all converging on the target point.
Perhaps of equal importance is the fact that increasing scientific evidence has persuaded the majority of the international neurosurgical community that radiosurgery is a viable treatment option for selected patients suffering from a variety of challenging neurosurgical disorders.

\section{Linear Accelerator-Based Radiosurgery Technique}

Although the details of radiosurgical treatment techniques differ somewhat from system to system, the basic paradigm is inclusive. We provide a detailed description of a typical radiosurgical treatment at the University of Florida.

Almost all radiosurgical procedures undertaken in adults are performed on an outpatient basis. The patient reports to the neurosurgical clinic the day before treatment to provide detailed history, undergo physical examination, and to be presented with an indepth review of the treatment options. If radiosurgery is deemed appropriate, the patient is sent to the radiology department where a volumetric MR imaging study is obtained. The next morning, the patient arrives at 7:00. A stereotactic head ring is applied after administration of a local anesthetic agent. No skin shaving or preparation is required. Subsequently, stereotactic CT scanning is performed. One-millimeter slices are obtained throughout the entire head. The patient is then transported to an outpatient holding area where he and his family have breakfast and relax until the treatment planning process is complete.

The volumetric MR imaging study obtained the previous day is used to generate a "preplan," which can be generated manually or by using completely automated program $^{58}$ that will place multiple isocenters in such a way as to generate an optimally conformal prescription isodose line (Fig. 2). On the day of treatment, the stereotactic CT scan is transferred via ethernet to the treatment-planning computer. Image fusion technology is used to fuse the stereotactic CT scan to the previously acquired MR images and, hence, to the previously generated plan. The preplan

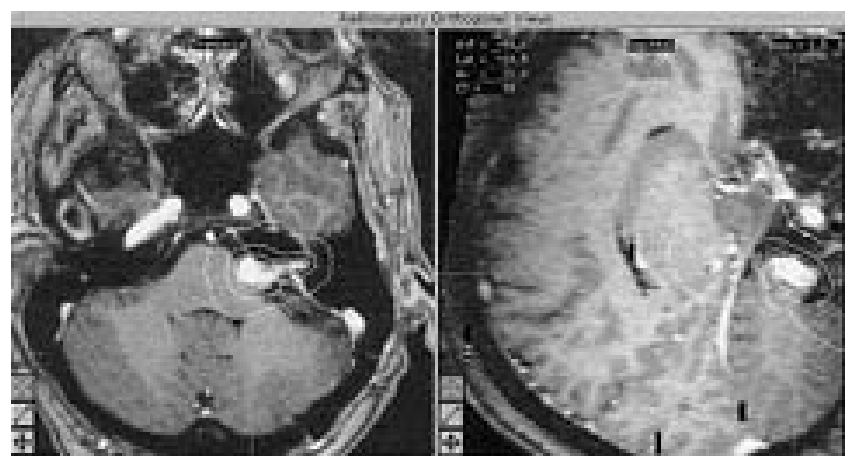

Fig. 2. Highly conformal dosimetry. Images of a VS shown with a six-isocenter treatment plan. The lines around the tumor, in centrifugal order, represent the 70, 35, and 14\% isodose lines. The prescription dose is delivered to the $70 \%$ isodose shell, which has been constructed, using various computer dose-planning tools, to conform closely to the surface of the tumor. Note that the $35 \%$ isodose line (by definition, the boundary at which the radiation dose has fallen to half of the prescription dose) lies within millimeters of the tumor margin. This steep dose gradient allows a high dose of radiation to be delivered to the tumor while exposing surrounding tissues to minimal radiation. 
is carefully examined and, if necessary, adjusted to generate the actual treatment plan.

When dose planning is complete, the radiosurgical device, (which improves the accuracy of the LINAC to $0.2 \mathrm{~mm}$ ), is attached to the LINAC. The patient then is attached to the device and treated. The head ring is removed, and after a short observation period, the patient is discharged. The radiosurgical device is disconnected from the LINAC, which is then ready for conventional usage. Close clinical and radiological neuroimaging follow-up examination is arranged at appropriate intervals depending on the entity treated and the condition of the patient.

\section{Radiosurgery for VS}

Among benign intracranial tumors, VS (acoustic neuroma) has, to date, been the most frequent target for stereotactic radiosurgery. This common lesion (representing $10 \%$ of all primary brain tumors) is a benign proliferation of Schwann cells arising from the myelin sheath of the vestibular branches of the eighth cranial nerve. These tumors are slightly more common in women, present at a mean age of 50 years, and occur bilaterally in patients with NF2.

Leksell ${ }^{28}$ first used stereotactic radiosurgery to treat a VS in 1969. Stereotactic radiosurgery is a logical alternative modality for this tumor for several reasons. A VS is typically well demarcated from surrounding tissues on neuroimaging studies. The sharp borders of this noninvasive tumor make it a convenient match for the characteristically steep dose gradient produced at the boundary of a radiosurgical target. This allows the radiosurgeon to minimize the radiation affecting the normal tissue. Excellent spatial resolution on Gd-enhanced MR images facilitates radiosurgical dose planning. These tumors typically occur in an older population of individuals who may be less fit for microsurgical resection requiring general anesthesia. Finally, the location of these tumors at the skull base in proximity to multiple critical neurological structures (that is, cranial nerves, brainstem) leads to appreciable surgeryinduced morbidity and rare cases of mortality even in expert hands. This makes the concept of an effective, less invasive, less morbid alternative treatment that could be performed in a single day and requires only a local anesthetic quite attractive. Whether radiosurgery fits this description has been extensively debated.

Certainly, the role of radiosurgery is limited by its inability to expeditiously relieve mass effect in patients for whom this is necessary. The radiobiological nature of stereotactic radiosurgery also requires lower, potentially less effective doses for higher target volumes to avoid complications. This limits its use of stereotactic radiosurgery to the treatment of smaller tumors. Despite these limitations, there is a growing body of evidence the literature that substantiates the claim that radiosurgery is a safe and effective alternative therapy for acoustic schwannomas. ${ }^{48}$

\section{Linear Accelerator-Based Series}

The published experience involving the use of LINACbased radiosurgery for the treatment of VSs is relatively limited compared with the GK literature. The authors of several reports have documented their early experience at University of Florida with this issue. ${ }^{38,39}$ Most recently,
Foote, et al., ${ }^{12}$ performed an analysis of risk factors associated with radiosurgery for VS. Their aim was to identify factors associated with delayed cranial neuropathy following radiosurgery for VS and to determine how such factors may be manipulated to minimize the incidence of radiosurgical complications while maintaining high rates of tumor control. Between July 1988 and June 1998, 149 patients with VS underwent LINAC-based radiosurgery at the University of Florida. In each case, the patient's tumor and brainstem were contoured in 1-mm slices on the original radiosurgical targeting images. Resulting tumor and brainstem volumes were coupled with the original radiosurgery plans to generate dose-volume histograms. Various tumor dimensions were also measured to estimate the length of cranial nerve that would be irradiated. Followup data, including evidence of cranial neuropathy and imaging-documented tumor control, were obtained from a prospectively maintained, computerized database. The authors conducted statistical analyses to compare the incidence of posttreatment cranial neuropathies or tumor growth among patient strata defined by risk factors of interest. One hundred thirty-nine of the 149 cases were included in the analysis of complications. The median duration of clinical follow up for this group was 36 months (range 18-94 months). The tumor control analysis included 133 cases. The median duration of radiological follow up in this group was 34 months (range 6-94 months). The overall 2-year actuarial incidences of facial and trigeminal neuropathies were 11.8 and $9.5 \%$, respectively. In 41 patients treated before 1994, the incidences of facial and trigeminal neuropathies were both $29 \%$, but in the 108 patients treated since January 1994 these rates declined to 5 and $2 \%$, respectively. An evaluation of multiple risk factor models showed that maximum radiation dose to the brainstem, treatment era (prior to 1994 compared with 1994 or later), and prior resection were all simultaneously informative predictors of cranial neuropathy risk. The radiation dose prescribed to the tumor margin could be substituted for the maximum dose to the brainstem with a small loss in predictive strength (Fig. 3). The overall radiological tumor control rate (Table 1) was $93 \%(59 \%$ of the tumors regressed, $34 \%$ remained stable, and $7.5 \%$ enlarged), and the 5-year actuarial tumor control rate was $87 \%$ (95\% confidence interval 76-98\%). Based on their findings in this study, the authors currently recommend administering a peripheral dose of $12.5 \mathrm{~Gy}$ for almost all acoustic lesions because this dose is most likely to yield long-term tumor control without causing cranial neuropathy.

Spiegelmann, et al., ${ }^{55,56}$ have reported their experience. They reviewed the methods and results of LINAC-based radiosurgery in 44 patients with acoustic neuromas treated between 1993 and 1997. Computerized tomography scanning was selected as the stereotactic imaging modality for target definition. A single conformally shaped isocenter was used in the treatment of 40 patients; two or three isocenters were used in four patients who harbored very irregularly shaped tumors. The radiation dose directed to the tumor border was the only parameter that changed during the study period. In the first 24 patients treated the dose was 15 to $20 \mathrm{~Gy}$, whereas in the more recently treated 20 patients the dose was reduced to 11 to 


\section{W. A. Friedman and K. D. Foote}

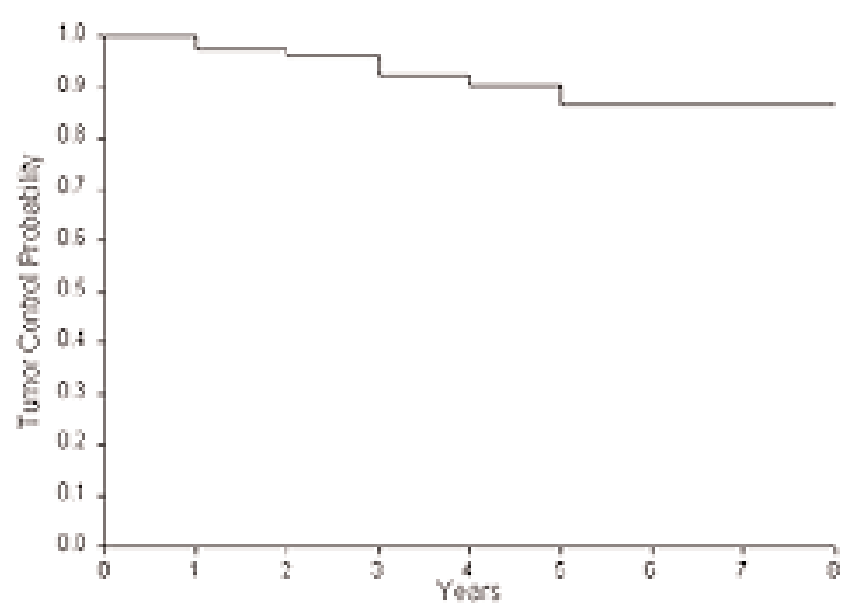

Fig. 3. Graph demonstrating actuarial tumor control reported for the University of Florida series.

$14 \mathrm{~Gy}$. After a mean follow-up period of 32 months (range 12-60 months), evidence of control was shown in $98 \%$ of the tumors. The actuarial hearing preservation rate was $71 \%$. New transient facial neuropathy developed in $24 \%$ of the patients and persisted to a mild degree in $8 \%$. The radiation dose correlated significantly with the incidence of cranial neuropathy, particularly in large tumors $\left(\geq 4 \mathrm{~cm}^{3}\right)$.

Several reports on smaller series of patients treated with LINAC-based radiosurgery for VSs have been published in recent years. Martens, et al. ${ }^{37}$ reported on 14 patients with at least 1 year of follow up after undergoing radiosurgery in a LINAC unit in the University Hospital in Ghent, Belgium. A mean marginal dose of 19.4 Gy (range 16-20 Gy) was delivered to the $70 \%$ isodose line with a single isocenter. The mean follow-up duration was 19 months (range 12-24 months). During this relatively short follow-up interval, $100 \%$ radiographic tumor control has been achieved (29\% of the lesions regressed, $71 \%$ stabilized, and none enlarged). Rates of delayed facial and trigeminal neuropathy were 21 and $14 \%$, respectively, and two of three facial nerve deficits resolved. Preoperative hearing was preserved $50 \%$ of the cases.

Valentino and Raimondi ${ }^{57}$ treated 23 patients with LINAC-based radiosurgery in Rome, Italy. Five suffered from NF and seven $(30 \%)$ had undergone previous surgery. The total radiation dose to the tumor margin ranged from 12 to $45 \mathrm{~Gy}$ (median $30 \mathrm{~Gy}$ ) and was delivered in one to five sessions. One or two isocenters were used and the mean follow-up duration was 40 months (range 2446 months). Results derived from this less conventional method of multisession radiosurgery were comparable with those of other radiosurgical techniques. Tumor control was achieved in $96 \%$ of patients (38\% of the lesion regressed, 58\% stabilized, and $4 \%$ enlarged), facial and trigeminal neuropathies each occurred at a rate of $4 \%$, and "hearing was preserved at almost the same level as that prior to radiosurgery in all patients."

The use of LINAC-based radiosurgery for acoustic neuromas is briefly discussed in reports by Delaney, et al., ${ }^{7}$ and Barcia, et al. ${ }^{2}$ In addition, fractionated stereotactic radiotherapy has been used as an alternative management for VSs. ${ }^{1,5}$ This method is proposed as a way of exploiting
TABLE 1

Incidence of cranial nerve palsy stratified by prescription dose in the University of Florida series

\begin{tabular}{lrrrr}
\hline \hline & & \multicolumn{3}{c}{ Cranial Nerve } \\
\cline { 3 - 5 } Radiation & No. of & VII & V & VII or VI (\%) \\
\hline Dose $(\%)$ & Cases & 0 & 0 & $0(0)$ \\
10 & 21 & 1 & 1 & $1(2.9)$ \\
12.5 & 35 & 10 & 9 & $14(22.6)$ \\
15 & 62 & 2 & 0 & $2(11.1)$ \\
17.5 & 18 & 2 & 2 & $2(100)$ \\
20 & 2 & 1 & 1 & $1(100)$ \\
22.5 & 1 & 16 & 13 & 20 \\
total & 139 & & & \\
\hline
\end{tabular}

the precision of stereotactic radiation delivery to minimize the dose to normal brain, while using lower fractionated doses in an effort to minimize complications. Thus far, most radiosurgeons believe that optimal results can be achieved using highly conformal single-fraction radiosurgery, while sparing the patient the inconvenience of a prolonged treatment course.

\section{Gamma Knife Series}

Following Leksell's initial report in 1971 on the treatment of three small VS with the GK at the Karolinska Institute in Stockholm, Hirsch, et al., ${ }^{19}$ reported on nine patients treated at the same facility between 1969 and 1974. This same group has periodically reported on their continuing experience. ${ }^{18,42,43}$ In 1993, Norén, et al., ${ }^{44}$ reported on 254 patients who underwent GK between 1969 and 1991. Of these, bilateral tumors were present in 61 patients with NF2. The minimum tumor dose ranged from 18 to 20 Gy specified at the 50 to $90 \%$ isodose line, the dose varying inversely with tumor size. This dose was reduced to 10 to 15 Gy during the final 3 years of the study. The minimum follow-up duration was 1 year (range 1-17 years). Rates of tumor control (decreased or stable tumor volume evidenced on neuroimaging) were $94 \%$ for unilateral tumors and $84 \%$ for those associated with NF2. Delayed facial and trigeminal neuropathies occurred in 17 and $19 \%$ of patients, respectively. These were predominantly transient deficits, but severe nerve injuries did occur in both the facial and trigeminal nerves at a rate of $4 \%$. These complication rates have diminished significantly recently with the institution of MR imaging-based dose planning and the use of lower radiation doses. Reportedly, the rates of facial and trigeminal nerve palsy for the most recently treated 55 patients were 1.8 and $0 \%$, respectively (a single mild facial paresis was observed).$^{41}$ Other complications included transient imbalance $(8 \%)$ and hydrocephalus (3\%). Of those patients with functional hearing prior to radiosurgery, only $22 \%$ had preserved hearing in the overall series. Remarkably, however, in a more recent analysis of the subset of 44 patients treated between 1991 and 1994 who had functional (Gardner- Robertson Class I or II) hearing prior to treatment, Norén reported the preservation of Class I or II hearing in $75 \%$ of patients after 1 year and $71 \%$ after 2 years. He attributed this improvement to better quality imaging and dose-planning accuracy that has resulted in more highly conformal radiation dose delivery. 
Researchers at the University of Pittsburgh have carefully documented their extensive experience with GKS for VSs during the past several years. ${ }^{9-11,22,29-34,45}$ In a recent update $^{33}$ the authors reported on 402 patients treated during a 10-year interval. Among these, 33 had NF2 and 97 (24\%) had previously undergone unsuccessful VS excision. Initially target definition was performed using CT scanning, but MR imaging replaced the latter over the past 5 years. The minimum dose to the tumor margin was 17 Gy during the early part of the series, but more recently doses of 12 to 14 Gy have been prescribed to at least the $50 \%$ isodose line. The mean follow-up duration was 36 months, and 88 patients underwent more than 7 years of follow up. Radiographic tumor control was achieved in $93 \%$ of patients $(30 \%$ of the lesions regressed, $63 \%$ stabilized, and $7 \%$ enlarged), but only $2 \%$ developed symptoms requiring surgical intervention. The complication rates diminished significantly in association with improvements in technique during the 10-year study interval. The incidence of delayed facial neuropathy was $28 \%$ when CT scanning-based dose planning was used, but this decreased to only $8 \%$ since the advent of MR imagingbased planning 5 years ago. Similarly, the rate of delayed trigeminal neuropathy was reduced from $34 \%$ when CT planning was used to $8 \%$ when MR imaging was instituted. The majority of these cranial neuropathies were transient. Other complications included transient imbalance $(5 \%)$ and hydrocephalus $(<3 \%)$. The percentage of patients in whom unchanged hearing was maintained in comparison with preoperative testing increased from 39\% with CT planning to $68 \%$ with MR imaging planning.

Also of particular interest is a recent publication by Kondziolka, et al., ${ }^{24}$ who reported long-term outcomes after radiosurgery in patients with acoustic neuromas. One hundred sixty-two patients underwent more than 5 years of careful follow up, and the rate of "tumor control"defined by the authors as cases in which the need for open resection was avoided-was $98 \%$ (62\% of the tumors regressed, $33 \%$ stabilized in size, and $6 \%$ grew); only four patients $(2 \%)$ required subsequent resection due to tumor progression. Although complication rates in this older series were predictably higher than those in more recent series, they were still relatively low. Normal facial nerve function was preserved in $79 \%$ of patients after 5 years, and normal trigeminal function was preserved in $73 \%$. In $51 \%$ of the patients no change in hearing ability occurred. Fifty-six percent of all neurological deficits resolved completely, and no new neurological deficits appeared more than 28 months after radiosurgery.

Foote, et al., ${ }^{13}$ initially reported on 36 patients who underwent GKS at the Mayo Clinic in 1993. In most cases a mean marginal dose of 16 to $20 \mathrm{~Gy}$ to the $50 \%$ isodose line was delivered. The mean follow-up duration was 16 months (range 2.5-36 months). Excellent tumor control $(100 \%)$ was reported, but the incidence of delayed cranial neuropathy was significantly higher than those in the aforementioned reports. Two-year actuarial incidences of facial and trigeminal neuropathy were 67 and 59\%, respectively. Hearing preservation occurred at a rate of $42 \%$. These higher complication rates have led the Mayo group to decrease the dose delivered to VSs and to avoid treating larger tumors. The group recently published a study comparing results obtained in their initial 42 patients with a series of 40 patients treated more recently with lower doses (12-16 Gy to the tumor margin, depending on tumor size).$^{14}$ Lower-dose radiation resulted in a reduced incidence of facial neuropathy from 38 to $8 \%$. Similarly, the rate of trigeminal neuropathy decreased from 29 to $15 \%$. Although the follow-up period was relatively short for these more recently treated patients, no tumor in the lower-dose group had shown progression.

The GKS group at the University of Virginia recently published a summary of their 10-year experience involving treatment of VSs. ${ }^{49}$ In their series, 153 of 200 treated patients were followed for a mean period of 4.3 years. Tumor volume ranged from 0.02 to $18.3 \mathrm{ml}$ (mean $2.8 \mathrm{ml}$ ) and patients were treated with doses ranging from 9 to 20 Gy (median $13 \mathrm{~Gy}$ ). The results were compared between two subsets of patients: 96 cases in which radiosurgery was the primary treatment and 57 cases in which prior microsurgical resection had failed. Tumor response was better in the former group, in which decreased volume was observed in $81 \%$, stabilization in $12 \%$, and increased volume in $6 \%$-compared with shrinkage in $65 \%$, stabilization in $25 \%$, and growth in $11 \%$ of the previously resected tumors. Complication rates were low, with only five patients $(3.3 \%)$ developing trigeminal dysfunction and three patients $(2 \%)$ developing facial weakness. Hearing deteriorated in $60 \%$ of patients.

A few smaller series of patients with VS treated with GKS have been reported recently. Ito, et al., ${ }^{21}$ reported on 46 patients treated with GKS unit at the University of Tokyo between 1990 and 1994. All dose planning was based on CT scanning data. A mean marginal dose of 17 Gy (range 12-25 Gy) was delivered to the 50\% isodose line. The mean follow-up duration was 39 months (range 4-73 months). Tumor control was achieved in $96 \%$ of cases (22\% of the lesions regressed, $74 \%$ stabilized, and $4 \%$ enlarged). Rates of delayed facial and trigeminal neuropathies were 22 and $30 \%$, respectively. Preoperative hearing was preserved in $39 \%$ of patients.

Kobayashi, et al. ${ }^{23}$ reported on 44 cases of VS treated at the Gamma Knife Center in Komaki City, Japan. A mean marginal dose of 14.8 Gy was delivered to the $50 \%$ isodose line. The mean follow-up duration was 12 months (range 3-20 months). One hundred percent tumor control was reported: neuroimaging-documented radiological regression occurred in $25 \%$ and stabilization occurred in $75 \%$ over this relatively short follow-up interval. Facial and trigeminal neuropathies developed at rates of 16 and $7 \%$, respectively. The majority of these cranial nerve deficits (four of seven facial and three of three trigeminal) resolved. Preoperative hearing was preserved in $48 \%$.

\section{THE SURGICAL ALTERNATIVE}

The mainstay of treatment for VS has long been resection, which has been significantly refined during the past 20 years. Results have been refined by use of the operating microscope, improved understanding of the involved microsurgical anatomy, modifications of the various approaches to the tumor site, and intraoperative neurophysiological monitoring of the facial and cochlear nerves. Recent reports by authors practicing at centers with extensive experience with surgical management of VSs confirm its efficacy and relatively low morbidity rate. 
Samii and Mathies ${ }^{51-53}$ and Samii and colleagues ${ }^{54}$ recently published a series of papers documenting excellent results after 1000 acoustic schwannoma resections. They achieved complete gross anatomical removal of the tumor in $98 \%$ of cases and reported a recurrence rate of only $1 \%$ in non-NF2 cases, although the follow-up duration was not indicated. Even in the hands of this master surgeon, however, the surgery-related morbidity and mortality rates were not negligible. Facial nerve continuity was lost in $7 \%$ of patients, and among the remaining $93 \%$ with anatomical preservation of the nerve, $45 \%$ suffered new facial deficits. Although many of these patients experienced improved facial function during the 1st postoperative year, House-Brackmann Grade IV or worse deficits were documented in $27 \%$ of all patients, giving them a high likelihood of permanent facial weakness. Other surgery-related complications included cerebrospinal fluid fistula (9\%), meningitis (3\%), intracranial hemorrhage (2\%), lower cranial nerve neuropathies $(2 \%)$, hemiparesis $(1 \%)$, quadriparesis in two patients $(0.2 \%)$, and death in 11 patients $(1 \%){ }^{36}$ Among those patients with hearing prior to surgery, $40 \%$ experienced preservation of some hearing postoperatively. In the treatment of their most recent 200 cases this rate has improved to $47 \%$ hearing preservation; however, the preservation rate of "useful hearing" (variably defined by different authors) was considerably lower and a function of tumor size and preoperative hearing quality. ${ }^{34}$

Gormley, et al., ${ }^{17}$ also reported outcomes in 179 patients with VSs who underwent surgery. In this series, complete gross anatomical resection was accomplished in $99 \%$, and the only recurrence occurred in the case in which the lesion was subtotally resected; the median follow-up period was 65 months. They categorized tumors as small $(<2$ $\mathrm{cm})$, medium $(2-3.9 \mathrm{~cm})$, and large $(\geq 4 \mathrm{~cm})$ and noted the relationship between tumor size and postoperative cranial nerve function. Surgery resulted in new facial neuropathies (House-Brackmann Grade $\geq$ III) in $4 \%$ of patients with small tumors, $26 \%$ with medium-sized tumors, and $62 \%$ of those with large tumors. Other reported complications include cerebrospinal fluid fistula (15\%), meningitis $(3 \%)$, lower cranial nerve neuropathy $(2 \%)$, cerebellar/brainstem injury with permanent ataxia in one patient $(0.6 \%)$, and death in two patients $(1.1 \%)$. Functional hearing preservation was achieved in $48 \%$ of cases involving small tumors, $25 \%$ of those involving mediumsized tumors, and in cases in which large tumors were resected.

Other authors have published similar surgery-related results for VSs. ${ }^{8,40}$ All of these reports have two factors in common: 1) they demonstrate the significant strides toward lower surgery-related morbidity and mortality rates that have been made in recent years, and 2) they represent optimal results obtained at centers with extensive experience in acoustic neuroma surgery. It is also worthwhile to note that patients generally report less satisfaction with their outcomes than what might be predicted based on reported similar surgery-related outcomes..$^{59}$

\section{CONCLUSIONS}

Based on our review, the following conclusions are made. 1) Long-term actuarial tumor control rates of greater than $90 \%$ are routinely reported after radiosurgery (Table 2). 2) The rates of trigeminal and facial nerve neuropathy after radiosurgery involving doses recommended for the past decade, are both less than 5\%. Most of these deficits are temporary. 3) Hearing preservation is greater than $50 \%$ in many radiosurgical series. 4) These results compare very favorably with the best neurosurgical series

TABLE 2

Reported results for VS radiosurgery

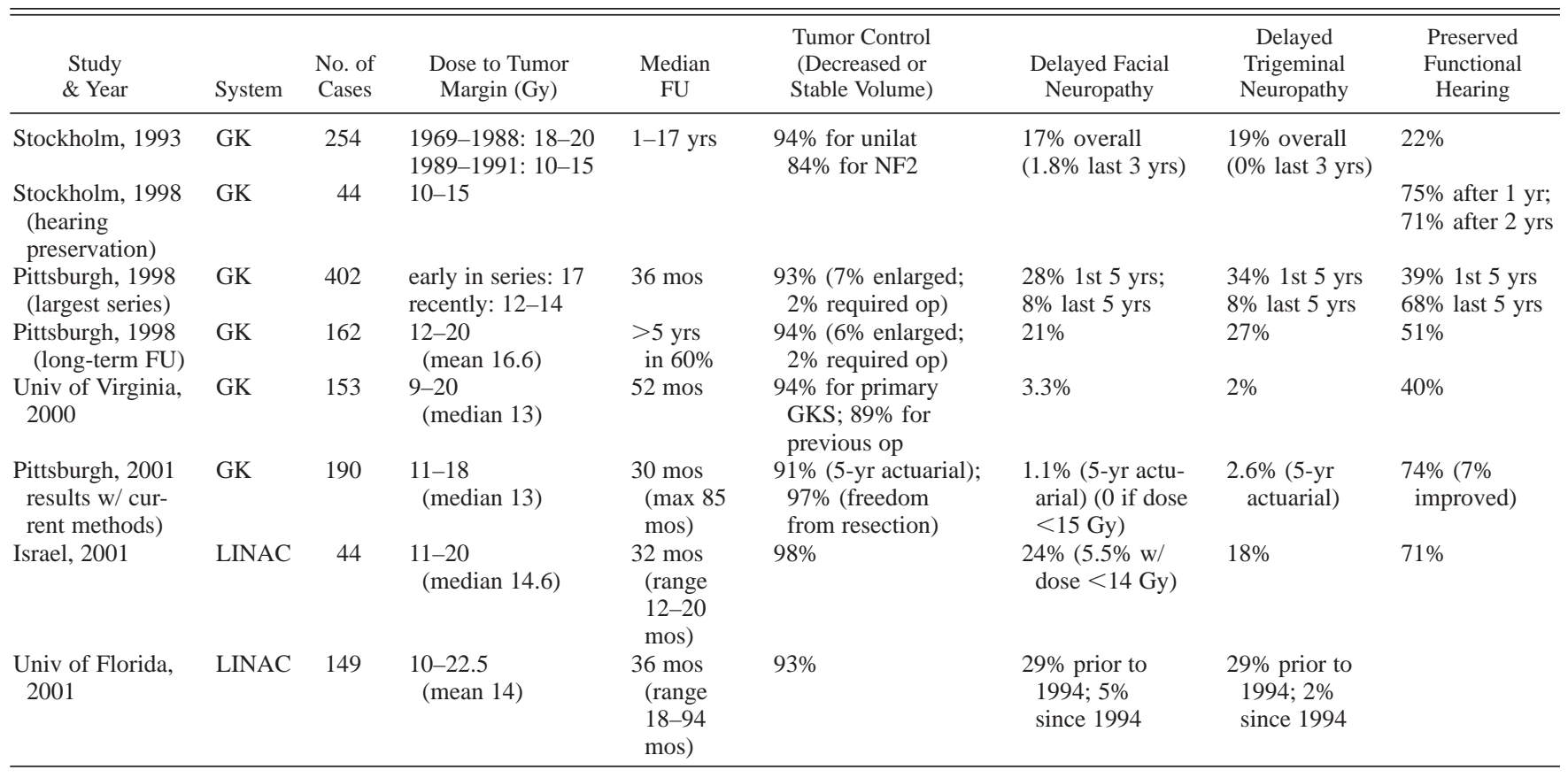


in the literature. 5) Radiosurgery is, therefore, the treatment of choice in cases of small VSs.

\section{References}

1. Andrews DW, Suarez O, Goldman HW, et al: Stereotactic radiosurgery and fractionated stereotactic radiotherapy for the treatment of acoustic schwannomas: comparative observations of 125 patients treated at one institution. Int $\mathbf{J}$ Radiat Oncol Biol Phys 50:1265-1278, 2001

2. Barcia Salorio JL, Hernandez G, Ciudad J, et al: Stereotactic radiosurgery in acoustic neurinoma. Acta Neurochir Suppl 33:373-376, 1984

3. Barrow DL, Bakay RA, Crocker I, et al: Stereotactic radiosurgery. J Med Assoc Ga 79:667-676, 1990

4. Betti OO, Derechinsky VE: Hyperselected encephalic irradiation with linear accelerator. Acta Neurochir Suppl 33: 385-390, 1984

5. Bush DA, McAllister CJ, Loredo LN, et al: Fractionated proton beam radiotherapy for acoustic neuroma. Neurosurgery 50: 270-275, 2002

6. Colombo F, Benedetti A, Pozza F, et al: Stereotactic radiosurgery utilizing a linear accelerator. Appl Neurophysiol 48: $133-145,1985$

7. Delaney G, Matheson J, Smee R: Stereotactic radiosurgery: an alternative approach to the management of acoustic neuromas. Med J Aust 156:440, 1992

8. Ebersold MJ, Harner SG, Beatty CW, et al: Current results of the retrosigmoid approach to acoustic neurinoma. J Neurosurg 76:901-909, 1992

9. Flickinger JC, Kondziolka D, Pollock BE, et al: Evolution in technique for vestibular schwannoma radiosurgery and effect on outcome. Int J Radiat Oncol Biol Phys 36:275-280, 1996

10. Flickinger JC, Lunsford LD, Coffey RJ, et al: Radiosurgery of acoustic neurinomas. Cancer 67:345-353, 1991

11. Flickinger JC, Lunsford LD, Linskey ME, et al: Gamma knife radiosurgery for acoustic tumors: multivariate analysis of four year results. Radiother Oncol 27:91-98, 1993

12. Foote KD, Friedman WA, Buatti JM, et al: Analysis of risk factors associated with radiosurgery for vestibular schwannoma. J Neurosurg 95:440-449, 2001

13. Foote R, Coffey R, Earle J, et al: Stereotactic radiosurgery using the gamma knife for acoustic neuromas. Proc ASTRO 35:151, 1993 (Abstract)

14. Foote RL, Coffey RJ, Swanson JW, et al: Stereotactic radiosurgery using the gamma knife for acoustic neuromas. Int J Radiat Oncol Biol Phys 32:1153-1160, 1995

15. Friedman WA, Bova FJ: The University of Florida radiosurgery system. Surg Neurol 32:334-342, 1989

16. Friedman WA, Buatti JM, Bova FJ: LINAC Radiosurgery - A Practical Guide. New York: Springer-Verlag, 1998

17. Gormley WB, Sekhar LN, Wright DC, et al: Acoustic neuromas: results of current surgical management. Neurosurgery 41:50-60, 1997

18. Hirsch A, Noren G: Audiological findings after stereotactic radiosurgery in acoustic neurinomas. Acta Otolaryngol 106: 244-251, 1988

19. Hirsch A, Noren G, Anderson H: Audiologic findings after stereotactic radiosurgery in nine cases of acoustic neurinomas. Acta Otolaryngol 88:155-160, 1979

20. Hitchcock E, Kitchen G, Dalton E, et al: Stereotactic LINAC radiosurgery. Br J Neurosurg 3:305-312, 1989

21. Ito K, Kurita H, Sugasawa K, et al: Analyses of neuro-otological complications after radiosurgery for acoustic neurinomas. Int J Radiat Oncol Biol Phys 39:983-988, 1997

22. Kamerer DB, Lunsford LD, Moller M: Gamma knife: an alternative treatment for acoustic neurinomas. Ann Otol Rhinol Laryngol 97:631-635, 1988
23. Kobayashi T, Tanaka T, Kida Y: The early effects of Gamma Knife on 40 cases of acoustic neurinoma. Acta Neurochir Suppl 62:93-97, 1994

24. Kondziolka D, Lunsford LD, McLaughlin MR, et al: Long-term outcomes after radiosurgery for acoustic neuromas. N Engl J Med 339:1426-1433, 1998

25. Kooy HM, Dunbar SF, Tarbell NJ, et al: Adaptation and verification of the relocatable Gill-Thomas-Cosman frame in stereotactic radiotherapy. Int J Radiat Oncol Biol Phys 30:685-691, 1994

26. Kyuma Y, Hayashi A, Kitamura T, et al: Stereotactic radiosurgery using a linear accelerator. Neurol Med Chir (Tokyo) 32:572-577, 1992

27. Leksell L: The stereotaxic method and radiosurgery of the brain. Acta Chir Scand 102:316-319, 1951

28. Leksell L: A note on the treatment of acoustic tumors. Acta Chir Scand 137:763-765, 1971

29. Linskey ME, Flickinger JC, Lunsford LD: Cranial nerve length predicts the risk of delayed facial and trigeminal neuropathies after acoustic tumor stereotactic radiosurgery. Int J Radiat Oncol Biol Phys 25:227-233, 1993

30. Linskey ME, Lunsford LD, Flickinger JC: Neuroimaging of acoustic nerve sheath tumors after stereotaxic radiosurgery. Am J Neuroradiol 12:1165-1175, 1991

31. Linskey ME, Lunsford LD, Flickinger JC: Radiosurgery for acoustic neurinomas: early experience. Neurosurgery 26: 736-744, 1990

32. Linskey ME, Lunsford LD, Flickinger JC: Tumor control after stereotactic radiosurgery in neurofibromatosis patients with bilateral acoustic tumors. Neurosurgery 31:829-839, 1992

33. Lunsford LD, Kondziolka D, Flickinger JC, et al: Acoustic neuroma management: evolution and revolution, in Kondziolka D (ed): Radiosurgery 1997. Basel: Karger, 1998, pp 1-7

34. Lunsford LD, Linskey ME: Stereotactic radiosurgery in the treatment of patients with acoustic tumors. Otolaryngol Clin North Am 25:471-491, 1992

35. Lutz W, Winston KR, Maleki N: A system for stereotactic radiosurgery with a linear accelerator. Int J Radiat Oncol Biol Phys 14:373-381, 1988

36. Maire JP, Caudry M, Darrouzet V, et al: Fractionated radiation therapy in the treatment of Stage III and IV cerebello-pontine angle neurinomas: long-term results in 24 cases. Int J Radiat Oncol Biol Phys 32:1137-1143, 1995

37. Martens F, Verbeke L, Piessens M, et al: Stereotactic radiosurgery of vestibular schwannomas with a linear accelerator. Acta Neurochir Suppl 62:88-92, 1994

38. Mendenhall WM, Friedman WA, Bova FJ: Linear acceleratorbased stereotactic radiosurgery for acoustic schwannomas. Int J Radiat Oncol Biol Phys 28:803-810, 1994

39. Mendenhall WM, Friedman WA, Buatti JM, et al: Preliminary results of linear accelerator radiosurgery for acoustic schwannomas. J Neurosurg 85:1013-1019, 1996

40. Nadol JB Jr, Chiong CM, Ojemann RG, et al: Preservation of hearing and facial nerve function in resection of acoustic neuroma. Laryngoscope 102:1153-1158, 1992

41. Norén G: Stereotactic radiosurgical treatment of acoustic neurinomas, in Lunsford LD (ed): Modern Stereotactic Neurosurgery. Boston: Martinus Nijhoff, 1998, pp 835-844

42. Norén G, Arndt J, Hindmarsh T, et al: Stereotactic radiosurgical treatment of acoustic neurinomas, in Lunsford LD (ed): Modern Stereotactic Neurosurgery. Boston: Martinus Nijhoff, 1988, pp 481-490

43. Norén G, Arndt J, Hindmarsh T: Stereotactic radiosurgery in cases of acoustic neurinoma: further experiences. Neurosurgery 13:12-22, 1983

44. Norén G, Greitz D, Hirsch A, et al: Gamma knife surgery in acoustic tumours. Acta Neurochir Suppl 58:104-107, 1993

45. Ogunrinde OK, Lunsford LD, Flickinger JC, et al: Stereotactic radiosurgery for acoustic nerve tumors in patients with useful 
preoperative hearing: results at 2-year followup examination. J Neurosurg 80:1011-1017, 1994

46. Pastyr O, Hartmann GH, Schlegel W, et al: Stereotactically guided convergent beam irradiation with a linear accelerator: localization-technique. Acta Neurochir 99:61-64, 1989

47. Patil AA: Adaptation of linear accelerators to stereotactic systems, in Lunsford LD (ed): Modern Stereotactic Neurosurgery Boston: Martinus Nijhoff, 1988, pp 471-480

48. Pollock BE, Lunsford LD, Kondziolka D, et al: Outcome analysis of acoustic neuroma management: a comparison of microsurgery and stereotactic radiosurgery. Neurosurgery 36: 215-229, 1995

49. Prasad D, Steiner M, Steiner L: Gamma surgery for vestibular schwannoma. J Neurosurg 92:745-759, 2000

50. Rocher FP, Sentenac I, Berger C, et al: Stereotactic radiosurgery: the Lyon experience. Acta Neurochir Suppl 63: 109-114, 1995

51. Samii M, Matthies C: Management of 1000 vestibular schwannomas (acoustic neuromas): hearing function in 1000 tumor resections. Neurosurgery 40:248-262, 1997

52. Samii M, Matthies C: Management of 1000 vestibular schwannomas (acoustic neuromas): surgical management and results with an emphasis on complications and how to avoid them. Neurosurgery 40:11-23, 1997

53. Samii M, Matthies C: Management of 1000 vestibular schwannomas (acoustic neuromas): The facial nerve-preservation and restitution of function. Neurosurgery 40:684-695, 1997

54. Samii M, Matthies C, Tatagiba M: Management of vestibular schwannomas (acoustic neuromas): auditory and facial nerve function after resection of 120 vestibular schwannomas in patients with neurofibromatosis 2. Neurosurgery 40:696-706, 1997

55. Spiegelmann R, Gofman J, Alezra D, et al: Radiosurgery for acoustic neurinomas (vestibular schwannomas). Isr Med Assoc J 1:8-13, 1999

56. Spiegelmann R, Lidar Z, Gofman J, et al: Linear accelerator radiosurgery for vestibular schwannoma. J Neurosurg 94: 7-13, 2001

57. Valentino V, Raimondi AJ: Tumour response and morphological changes of acoustic neurinomas after radiosurgery. Acta Neurochir 133:157-163, 1995

58. Wagner TH: Optimal delivery techniques for intracranial stereotactic radiosurgery using circular and multileaf collimators. Dissertation. Gainsville: University of Florida, 2000.

59. Wiegand DA, Fickel V: Acoustic neuroma - the patient's perspective: Subjective assessment of symptoms, diagnosis, therapy, and outcome in 541 patients. Laryngoscope 99:179-187, 1989

60. Winston KR, Lutz W: Linear accelerator as a neurosurgical tool for stereotactic radiosurgery. Neurosurgery 22:454-464, 1988

Manuscript received March 5, 2003.

Accepted in final form April 14, 2003.

Address reprint requests to: William A. Friedman, M.D., Department of Neurosurgery, P.O. Box 100265, UFBI, University of Florida, Gainesville, Florida 32610. e-mail: friedman@ neurosurgery.ufl.edu. 